\title{
Seasonality in Multiple Maternities
}

\author{
Johan Fellman \\ Hanken School of Economics, Helsinki, Finland
}

\begin{abstract}
In the 19th century, a series of international statistical congresses introduced common rules for the national demographic registers. This activity contributed to the genesis of statistical research. During the history of twin research, Hellin's law has played a central role because it is an approximately correct association between the rates of multiple maternities. However, it has been mathematically proven that Hellin's law cannot hold exactly. The majority of all studies of Hellin's law are based on empirical rates of multiple maternities. Such studies can never confirm the law, but only identify errors too large to be characterized as random. It is of particular interest to examine why the rates of higher multiple maternities are sometimes too high or too low when Hellin's law is used as a benchmark. However, divergences from the law are often difficult to explain and/or eliminate. Different improvements to the law have been proposed. In this article, we study the seasonality of multiple maternities. We apply Hellin's law to compare the seasonality of twin and triplet rates.
\end{abstract}

Keywords: dizygotic, monozygotic, Hellin's law, seasonality, sinusoidal models, trigonometric regression models, variable transformation, history of twin research

In the 19th century, a series of statistical congresses underlaid demographic studies, including twin research. Levi (1854) gave a detailed presentation of the suggestions concerning the annual registry of populations proposed in 1853 at the Brussels congress. According to Brown (1872), at the congress held in 1872 in St. Petersburg, the principal discussion was related to the movements of the population and the mode in which they should be registered. Later, Westergaard (1932) devoted an entire chapter to the presentation of the statistical congresses of the 19th century.

Hellin's (1895) paper can be considered a milestone in the history of twin research, and one can divide the research into studies before and after Hellin. Spengler (1848) presented birth data, including data concerning multiple births for Mecklenburg-Schwerin for the period 1777-1847. Veit (1855) published a data set from Prussia (1826-1849), but in these studies there were no discussions concerning the relation between the number of twin and triplet maternities. Bertillion (1898) considered multiple maternity data from different countries in Europe. He foreboded Hellin's law because he presented the number of twin maternities in relation to one triplet maternity. In 1877, Neefe published his classical work (Neefe, 1877). He focused on the importance of the standardization of the demographic registers in different countries and used the new possibilities that the improved birth registers offered. Although other contemporaneous studies were published, our view is that the history of twin research starts from his publication because he analyzed a long series of problems connected to twinning. These have been shown to be central in later studies. It is important to note that Neefe did not anticipate Hellin's law.

Strassmann (1889) analyzed data published by Veit (1855) concerning single and higher maternities. Strassmann noted that there is one twin maternity per 89 and one triplet maternity per $(89)^{2}$ total maternities. Drejer (1895) was apparently not aware of Hellin (1895), but referred to Strassmann (1889) and stated that Strassmann had noted the relation between the rates of twin and triplet maternities (Fellman \& Eriksson, 2009).

Hellin (1895) observed an empirical relationship between the rates of twins and triplets. He stated that among human beings there is on average one twin maternity per 89 singleton maternities, one triplet maternity per $(89)^{2}$ singleton maternities, and in general, within the range of the possibility, one $\mathrm{x}$-tuplet maternity per $(89)^{\mathrm{x}-1}$ singleton maternities. Strassmann related the number of multiple maternities to the number of all maternities, while Hellin related the number of multiple maternities to the number of single maternities. However, both used the same relation: 1:89.

RECEIVED 4 September 2017; ACCEPTED 11 September 2017. First published online 12 October 2017.

ADDRESS FOR CORRESPONDENCE: Johan Fellman, Hanken School of Economics, PO Box 479, FI-00101 Helsinki, Finland. E-mail: fellman@hanken.fi 
Different studies of the seasonality of multiple maternities have been presented. James (1980) compared the seasonality of twin and triplet maternities. Bonnelykke et al. (1987) constructed seasonal models for twin maternities and included both temporal and seasonal components in the models. They included the temporal component because the twinning rate (TWR) showed strong temporal variations. In addition, they made use of the fact that the gestation for twin maternities is on average shorter than for singleton maternities. Elster and Bleyl (1991) studied the seasonality of triplet maternities. They published 1,050 triplet maternities for the period 1985-1988. These triplet data were compared with the natality statistics for the United States during the overlapping 4-year period in 1983-1986. Statistics for the exact comparison period were not published when Elster and Bleyl performed their study, so the closest complete overlapping 4-year period (19831986) was used instead of the exact one. Elster and Bleyl ignored the difference between the two periods and gave arguments for this. Furthermore, they did not present twinning data.

\section{Methods and Materials}

A problem that aggravates the discussion of Hellin's law is that the law is a mathematical rule concerning the theoretical rates, but all checks of the law are based on empirically obtained rates, and no exact proof to support the law can be obtained. In fact, one can only check whether observed inconsistencies are too large to be explained as random errors. In other studies, we have evaluated the association between the TWR, the triplet rate (TRR), and the Hellintransformed triplet rate (HRR) when the data are grouped according to time (Fellman, 2017b).

In this study, we split the data according to season. When the seasonality is studied, one must distinguish between two alternative problems: (a) the general significant heterogeneity - for example, tested by the $\chi^{2}$ test given below; or (b) the application of specific seasonal models discussed later.

\section{Seasonality Tests}

Eriksson and Fellman (2000) stressed that if one intends to identify the seasonality of multiple maternities, one must consider the seasonality of the corresponding rates. Doing this, the seasonality of the total maternities is eliminated.

Fellman (2017a) tested the seasonality of births by $\chi^{2}$ tests. Let $N_{\mathrm{i}}$ be the observed number of births in month number $i$ and let $k_{i}$ be the length of the month in days. Furthermore, let $N=\sum_{i=1}^{12} N_{i}$ be the total number of births and let $k=\sum_{i=1}^{12} k_{i}$ be the length of the year. The expected number of births per day for the whole year is $\frac{N}{k}$ and in month number $i$ it is $\hat{N}_{i}=k_{i} \frac{N}{k}$. The $\chi^{2}$ test is

$$
\chi^{2}=\sum_{i=1}^{12} \frac{\left(N_{i}-\hat{N}_{i}\right)^{2}}{\hat{N}_{i}}
$$

with 11 degrees of freedom.

For twin and other multiple maternities, we must modify the test. For instance, for twin sets, let $n_{i}$ be the number of twin maternities in month number $I$, and let $N$ be the total number of twin maternities. The total TWR is $r=\frac{n}{N}$. The observed TWR for month $i$ is $r_{i}=\frac{n_{i}}{N_{i}}$. The expected number of twin maternities in month number $i$ is $\hat{n}_{i}=r N_{i}$. Hence, the $\chi^{2}$ test for the twin data is

$$
\chi^{2}=\sum_{i=1}^{12} \frac{\left(n_{i}-\hat{n}_{i}\right)^{2}}{\hat{n}_{i}}
$$

with 11 degrees of freedom. A similar formula holds for higher multiple maternities and especially in this study for TRR. The critical values for $\chi^{2}$ tests with 11 degrees of freedom are $\chi^{2}=19.68$ for $p<.05, \chi^{2}=24.73$ for $p<.01$, and $\chi^{2}=31.26$ for $p<.001$.

\section{Seasonality Models}

If one intends to obtain a seasonal model, the simplest model could be the sinusoidal one:

$$
\begin{aligned}
R\left(t_{i}\right) & =K+R \sin \left(t_{i}+\alpha\right)+\varepsilon_{i} \\
& =K+A \cos \left(t_{i}\right)+B \sin \left(t_{i}\right)+\varepsilon_{i},
\end{aligned}
$$

where $A=R \sin \alpha, B=R \cos \alpha$, and the error terms $\varepsilon_{\mathrm{i}}$ are assumed to be independent and homoscedastic. However, it will be seen in many situations that although there are marked seasonal variations, the simple sine curve does not fit the data. For example, this is the case when the data show more than one marked peak. Data showing one peak and one trough may also differ markedly from the sine curve. The trough or the peak may be too long or the time between them may not be 6 months.

If one intends to obtain an improved seasonal model, it could be the trigonometric regression model (Fellman \& Eriksson, 2002):

$$
\begin{aligned}
R\left(t_{i}\right) & =K+\sum_{m=1}^{M} R_{m} \sin \left(m t_{i}+\alpha_{m}\right)+\varepsilon_{i} \\
& =K+\sum_{m=1}^{M}\left(A_{m} \cos \left(m t_{i}\right)+B_{m} \sin \left(m t_{i}\right)\right)+\varepsilon_{i}
\end{aligned}
$$

where $M$ is the number of pairs of trigonometric terms, $A_{m}=R_{m} \sin \alpha_{\mathrm{m}}$ and $B_{m}=R_{m} \cos \alpha_{\mathrm{m}}(m=1, \ldots, M)$. The error terms $\varepsilon_{\mathrm{i}}$ are assumed to be independent and homoscedastic. With monthly data, one has to introduce the restriction $M \leq 5$.

The parameters $A_{m}$ and $B_{m}(m=1, \ldots, M)$ and $K$ are estimated by ordinary least squares for the monthly 
data and the initial parameters $\alpha_{m}$ and the amplitudes $R_{m}=\sqrt{A_{m}^{2}+B_{m}^{2}}(m=1, \ldots, M)$ can be estimated by the equations:

$$
\tan \left(\hat{\alpha}_{m}\right)=\frac{\hat{A}_{m}}{\hat{B}_{m}}
$$

and

$$
\hat{R}_{m}=\sqrt{\hat{A}_{m}^{2}+\hat{B}_{m}^{2}} .
$$

The angle $\alpha_{m}$ and the amplitudes $R_{m}$ have to be estimated from formulae (5) and (6) irrespective of the significance of the parameter estimates. Therefore, Fellman and Eriksson (2002) recommended full pairs of trigonometric terms. The argument for this is that $\hat{R}_{m}$ may differ significantly from zero, but the angle $\alpha_{m}$ may be such that $\hat{A}_{m}$ or $\hat{B}_{m}$ is close to zero and consequently non-significant. From this, it follows that the tests of parameter significance should be applied to $\alpha_{m}$ and $\hat{R}_{m}(m=1, \ldots, M)$, but not to $\hat{A}_{m}$ or $\hat{B}_{m}$.

Fellman and Eriksson (2002) assumed that the model is optimal when the adjusted coefficient of determination, $\bar{R}^{2}$, attains its maximum. If $\bar{R}^{2}$ increased monotonically with increasing $M$, they chose $M=4$. In doing so, they left three degrees of freedom for the testing. The multiple trigonometric regression models and the corresponding tests of the estimates are discussed in detail in Fellman and Eriksson (2000) and in some of the references given in that article.

In this study, we perform an alternative attempt, that is, we pursue optimal seasonal models before we compare the obtained models for the different rates, and therefore, we concentrate on the individual parameters $\left\{A_{m}\right\}$ and $\left\{B_{m}\right\}$. Hence, we include in the models all significant parameters and ignore the insignificant ones.

The triplet sets are very rare and consequently suitable data containing sufficiently large sets for statistical analyses of monthly triplet births are difficult to obtain. James (1980) had the opportunity to perform his studies based on data from England and Wales (1952-1975). He compared the seasonality of opposite-sex twin sets, monozygotic twin sets, and triplet sets. His findings are presented in the Discussion section. In this study, we base our analyses on James' data and our central topic is to compare the seasonality of TWR, TRR, and HRR.

Elster and Bleyl (1991) published data about 1,050 triplet maternities for the period 1985-1988. These triplet data were compared with the natality statistics for the United States during the overlapping 4-year period in 1983-1986. They ignored the difference between the two periods and gave arguments for this. In our analyses, we accept the data and rules proposed by Elster and Bleyl.

\section{Results}

\section{Seasonal Heterogeneity}

We start with the tests of the seasonality. For James' data, we obtain $\chi^{2}=30632.24$ for the total births, $\chi^{2}=53.17$ for
TWR, and $\chi^{2}=16.31$ for TRR. Hence, for the total number of births and the TWR the test values are significant $(p<.001)$, but for the TRR it is non-significant $(p>.05)$. Although the TRR shows marked variations, the data set is too small for significance.

The available data in Elster and Bleyl yield only approximate triplet rates. We accept the assumptions of Elster and Bleyl and approximate the TRR by assuming that the data are constant during the whole period in 1983-1988. For the Elster and Bleyl data, we obtain $\chi^{2}=19472.94$ for the total births and $\chi^{2}=7.94$ for TRR. Hence, for the total number of births the test value is significant $(p<.001)$, but for the TRR, showing marked variations, it is non-significant $(p>$ .05 ) because the triplet data set is too small.

To compare the seasonality in more detail, we standardized the total number of births, TWR, TRR, and HRR into monthly indices having means of 100 (Eriksson \& Fellman 1999, 2000; Fellman \& Eriksson, 1999). Statistical comparisons using standard deviations (SDs) are applicable when indices are studied. If one tests seasonal variations in the indices, one obtains the SDs 4.590 for total births, 10.067 for TRR, 5.062 for HRR, and 1.802 for TWR. The analyses of James' data that reveal obvious discrepancies between TWR and $\mathrm{HRR}$ are obvious. If one tests seasonal variations in the indices for the Elster and Bleyl data, one obtains 3.78 for total births, 9.16 for TRR, and 4.508 for HRR. When we combine these findings, our conclusion is that all rates show seasonality. Furthermore, the results indicate the strongest seasonality in TRR, strong seasonality in the births and HRR, and only slight seasonality in TWR.

\section{Trigonometric Regression Models}

When we perform the proposed regression analyses, we obtain regression models for births, TWR, TRR, and HRR based on James' data for England and Wales. The goodness of fit is strongest for the birth data, medium for TWR and TRR and slight for HRR (Table 1). For the U.S. data given by Elster and Bleyl, we consider births, TRR, and HRR. Twinning data were not available in the U.S. data. The best fit is observed for births, but slight goodness of fit is observed for TRR and HRR (Table 1).

In Figure 1, we present the seasonality of TWR, TRR, and HRR based on James' data for England and Wales (1952-1975). One observes that the seasonal fluctuations are strongest for TRR, and for TWR and HRR the seasonality is of similar strength. The discrepancies between the seasonality pattern of TWR, TRR, and HRR are obvious.

In Figure 2, we present the seasonality of births, TRR, and HRR based on U.S. data (1983-1988). The births display the best goodness of fit (Table 1). One observes that the seasonal fluctuations are strongest for TRR. For births and HRR, the seasonality is of similar strength. The discrepancies between the seasonality pattern of births, TRR, and HRR are obvious. 
TABLE 1

Trigonometric Regression Models Including the Adjusted Coefficients of Determination, $\bar{R}^{2}$ as Goodness of Fit Measure

\begin{tabular}{|c|c|c|c|}
\hline Variables & Models & $\bar{R}^{2}$ & Data set \\
\hline Birth & Births $=99.977-1.985 \cos (t)+5.163 \sin (t)-1.607 \cos (2 t)+1.600 \sin (2 t)$ & 0.885 & James (1980) \\
\hline TWR & TWR $=100.054+1.936 \cos (t)-0.848 \sin (t)$ & 0.691 & James (1980) \\
\hline TRR & $\begin{array}{l}\text { TRR }=99.978+9.007 \cos (t)+4.094 \sin (t) \\
4.271 \sin (2 t)-4.165 \sin (3 t)-3.869 \sin (4 t)+5.193 \cos (5 t)\end{array}$ & 0.871 & James (1980) \\
\hline HRR & $\mathrm{HRR}=99.854+4.508 \cos (t)$ & 0.375 & James (1980) \\
\hline Births & Births $=100.002-3.205 \cos (t)-3.038 \sin (t)-0.919 \cos (2 t)+2.148 \sin (2 t)-0.749 \sin (4 t)-0.696 \sin (5 t)$ & 0.973 & Elster and Bleyl (1991) \\
\hline TRR & $\mathrm{TRR}=100.072+6.489 \sin (t)-6.864 \sin (2 t)$ & 0.484 & Elster and Bleyl (1991) \\
\hline
\end{tabular}

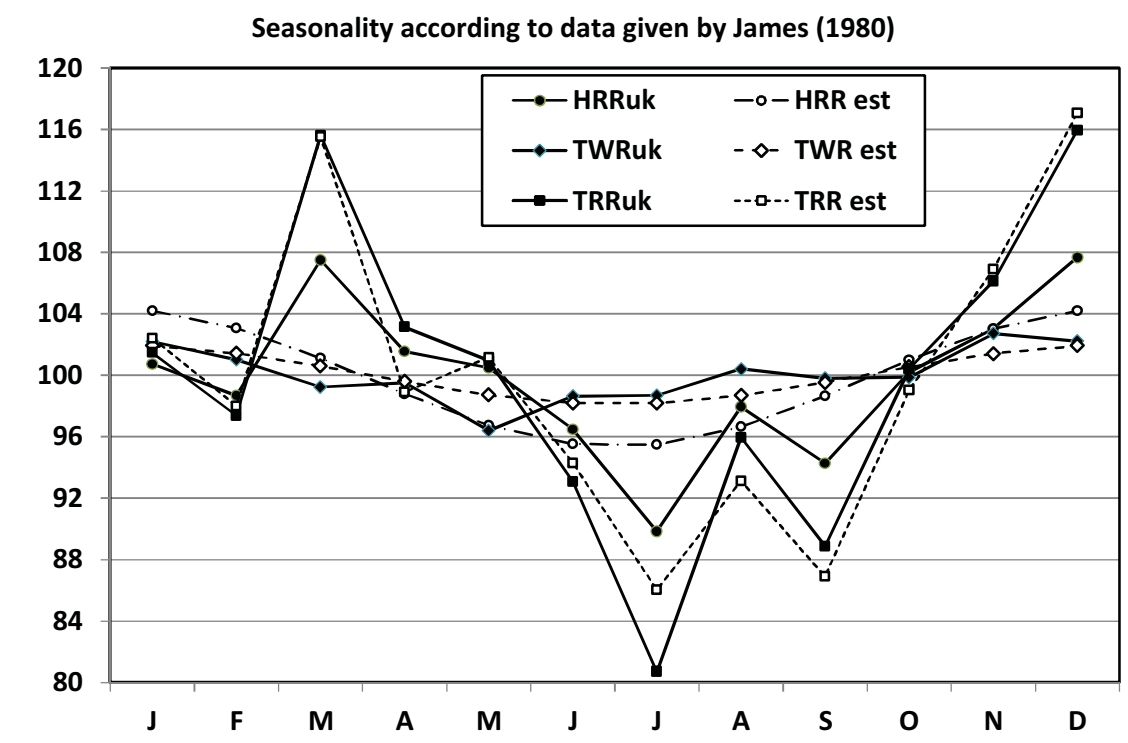

\section{FIGURE 1}

(Colour online) Comparison of the seasonality of twinning rate (TWR), triplet rate (TRR), and Hellin-transformed TRR (HRR) based on James' data for England and Wales (1952-1975). The analysis is based on transformed indices with means of 100. The obtained regression models are given in Table 1, and the seasonal variations are discussed in the text.

Seasonality in the US according to data given by Elster \& Bleyl (1991)

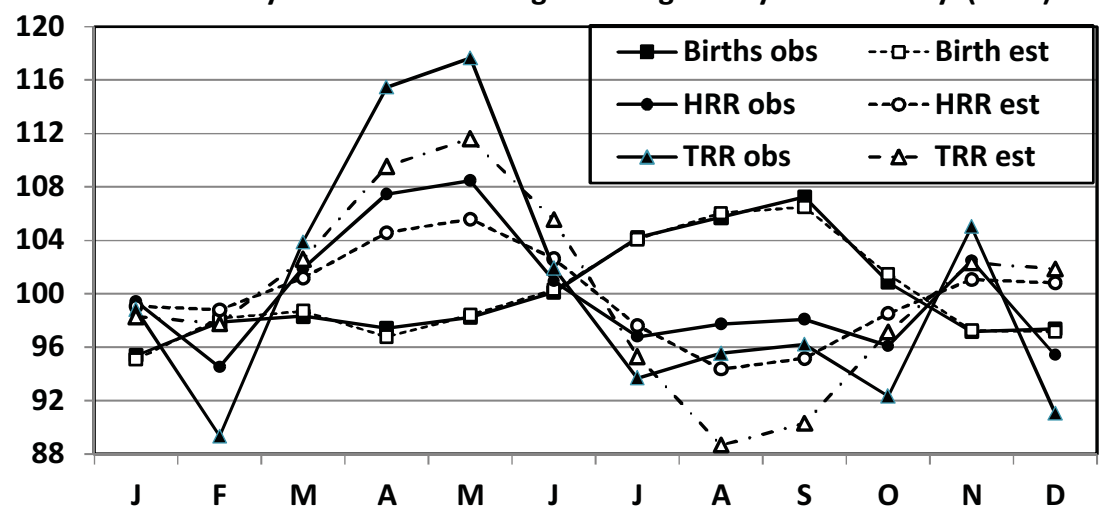

\section{FIGURE 2}

(Colour online) Comparison of the seasonality of total births (Births), TRR, and HRR for the United States (1983-1988). The analysis is based on the Elster and Bleyl data transformed to indices with means of 100. The obtained regression models are given in Table 1, and the seasonal variations are discussed in the text. 


\section{Discussion}

It is commonly agreed that the main argument for Hellin's law is that the probabilities of additional ovulations and the fissions of fertilized eggs can be explained by stochastic models (see, e.g., Allen \& Firschein, 1957; Fellman \& Eriksson, 2009; Jenkins, 1927; 1929; Jenkins \& Gwin, 1940; Zeleny, 1921). Hence, in large data sets the averages could be stable and formulated by a mathematical relation (Hellin's law).

Zeleny (1921) discussed in a short note Hellin's law. He stated that from the statistical relations it would appear that triplets are produced by the coincidence of two independent processes occurring with equal frequencies. One of these processes by itself gives rise to twin pairs. This relation would apply to any mode of origin of multiple births or to different combinations of these provided that each followed the rule. In fact, Zeleny considered the Strassmann version that the rates are related to the total number of maternities. He also referred to the Veit (1855) data and found startlingly good agreement with the law. Zeleny's analyses yielded him the honor that some authors later renamed as the HellinZeleny law. In addition, the arguments for the discrepancies are that after the fertilizations there is a long process influenced by disturbing factors. For example, Jenkins (1927) and Komai and Fukuoka (1936) assumed that differential mortality of twins and triplets in utero could be a disturbing factor. Consequently, the final result shows only a weak resemblance with the outcome of a stochastic process. This seems to be one main cause of the discrepancies between Hellin's law and the empirical findings. Among other issues the proposed model may be too simple.

Already, Jenkins (1927) noted that Hellin's law can be assumed only for maternal age-specific disaggregated data and that the discrepancy between Hellin's law and the observed aggregated data demands the use of the formula:

$$
T R R=\frac{1}{n} \sum_{i}\left(T W R_{i}\right)^{2} n_{i} .
$$

Fellman and Eriksson (1993) gave a mathematical proof that Hellin's law cannot hold exactly. If one aggregates heterogeneous data, the fluctuations are smoothed out, but the relation between the TWR and the TRR is not linear, and consequently, the aggregated and disaggregated data cannot simultaneously satisfy Hellin's law. In fact, Jenkins' arguments coincide mathematically with the integral proposed by Fellman and Eriksson (1993). However, Jenkins did not explain the disagreement between Hellin's law for aggregated and disaggregated data.

James (1980) observed that the TRR had a stronger seasonality than the TWR s (both the opposite-sex TWR and the monozygotic TWR). He found that there was good evidence that the incidence of opposite-sex twin births was seasonal. Triplet births seemed to show a seasonal variation at the same location and with double the amplitude.
He stated that this seasonal variation of polyzygotic maternities was due to seasonal variation in the rates of multiple ovulations. Of importance is James' remark that the monozygotic rate (MZR) and the dizygotic rate (DZR) are not observed rates, but estimated ones. Hence, the variance formulae given for observed rates are incorrect for these estimated rates. Following a different route, Fellman (2013) later found similar results and presented exact alternative formulae for the SDs of the MZR and DZR. Elster and Bleyl (1991) noted seasonality in the number of triplet births and that its pattern differed markedly from the seasonality of the births of the entire U.S. population (cf. Figure 2).

The history of the studies of Hellin's law shows a long series of attempts to reduce the discrepancies between the observed data and Hellin's law. Some studies based on MZR and DZR give improved results, but cannot eliminate the obtained discrepancies in the data (Allen, 1960; Bulmer, 1970; Fellman \& Eriksson, 2004). In conclusion, one can note that every 'improved' model has the same weakness as Hellin's law. The models are mathematically exact, but the analyses are based on empirical data. Hence, the question remains of whether the improvements are general or specific to the data considered.

\section{Acknowledgments}

We are grateful to the personnel at The National Library of Health Sciences at the University of Helsinki (Terkko) for providing us - my late friend and colleague Aldur W. Eriksson and myself - with copies of old publications concerning twin studies in the 19th century, which are otherwise difficult to obtain.

\section{References}

Allen, G. (1960). A differential method for estimation of type frequencies in triplets and quadruplets. American Journal of Human Genetics, 12, 210-224.

Allen, G., \& Firschein, I. L. (1957). The mathematical relations among plural births. American Journal of Human Genetics, 9, 181-190.

Bertillion, J. (1898). La gémellité selon l'age de la mére et le rang chronologique de l'accouchement (1). Journal de la Société de Statistique de Paris, 38, 146-152.

Bonnelykke, B., Søgaard, J., \& Nielsen, J. (1987). Seasonality in twin birth rates, Denmark, 1936-84. Journal of Epidemiology and Community Health, 41, 338-343.

Brown, S. (1872). Report on the eighth international statistical congress held at St. Petersburg, 22nd/10th August to $29 \mathrm{th} / 17 \mathrm{th}, 1872$. Journal of the Statistical Society of London, 35, 431-457.

Bulmer, M. G. (1970). The biology of twinning in man. Oxford, UK: Oxford University Press. 
Drejer, P. (1895). Om tvillinger [About twins]. Tillægshefte til 'Norsk Magazin for lægevidenskaben'. Kristiania.

Elster, A. D., \& Bleyl, J. (1991). Seasonality of triplet births in the United States. Human Biology, 63, 711-718.

Eriksson, A. W., \& Fellman, J. (1999). Seasonal variations of twin maternities in Denmark: Secular and regional differences. In Proceedings of the annual conference of the australasian society for human biology and the 5th world academic conference on human ecology of the IUAES's commission of human ecology. Adelaide, Australia, 1-5 December, 1997. Perspectives in Human Biology, 4, 213-221.

Eriksson, A. W., \& Fellman, J. (2000). Seasonal variation of livebirths, stillbirths, extramarital births and twin maternities in Switzerland. Twin Research, 3, 189-201.

Fellman, J. (2013). Statistical analyses of monozygotic and dizygotic twinning rates. Twin Research and Human Genetics, 16, 1107-1111.

Fellman, J. (2017a). Studies of the seasonal pattern of multiple maternities. Twin Research and Human Genetics, 20, 250256.

Fellman, J. (2017b). Aspects on the history of twin research: Statistical congresses in the 19th century and Hellin's law. Manuscript in preparation.

Fellman, J. O., \& Eriksson, A. W. (1993). Biometric analysis of the multiple maternities in Finland, 1881-1990 and in Sweden since 1751. Human Biology, 65, 463-479.

Fellman, J., \& Eriksson, A. W. (1999). Secular changes in the seasonal patterns of births in Nordic countries. In Proceedings of the annual conference of the Australasian society for human biology and the 5th world academic conference on human ecology of the IUAES's commission of human ecology. Adelaide, Australia, 1-5 December, 1997. Perspectives in Human Biology, 4, 203-212.

Fellman, J., \& Eriksson, A. W. (2000). Statistical analysis of the seasonal variation in demographic data. Human Biology, 72, 851-876.

Fellman, J., \& Eriksson, A. W. (2002). Regional, temporal and seasonal variations in births and deaths: The effects of famines. Social Biology, 48, 86-104.

Fellman, J., \& Eriksson, A. W. (2004). Association between the rates of multiple maternities. Twin Research, 7, 387-397.
Fellman, J., \& Eriksson, A. W. (2009). On the history of Hellin's law. Twin Research and Human Genetics, 12, 183-190.

Hellin, D. (1895). Die ursache der multiparität der uniparen tiere überhaupt und der zwillingsschwangerschaft beim menschen insbesondere. München, Germany: Seitz \& Schauer.

James, W. H. (1980). Seasonality in twin and triplet births. Annals of Human Biology, 7, 163-175.

Jenkins, R. L. (1927). The interrelations of the frequencies of plural births. Journal of Heredity, 8, 387-394.

Jenkins, R. L. (1929). Twin and triplet birth ratios. A further study of the interrelations of the frequencies of plural births. Journal of Heredity, 20, 485-494.

Jenkins, R. L., \& Gwin, J. (1940). Twin and triplet births ratios. Journal of Heredity, 31, 243-248.

Komai, T., \& Fukuoka, G. (1936). Frequency of multiple births among the Japanese and related people. American Journal of Physical Anthropology, 21, 433-447.

Levi, L. (1854). Resume of the Statistical Congress, held at Brussels, September 11th, 1853, for the purpose of introducing unity in the statistical documents of all countries. Journal of the Statistical Society of London, 17, 1-14.

Neefe, M. (1877). Zur Statistik der Mehrgeburten [Statistics of multiple maternities]. Jahrbücher für Nationalökonomie und Statistik, 28, 168-194.

Spengler, L. (1848). Statistische Uebersicht der seit 71 Jahren in den Mechlenburg-Schwerin's chen Landen vorgekommenen Geburten und der Sterblichkeit im Wochenbette. Neue Zeitschrift für Geburtskunde, 25, 439-446.

Strassmann, P. (1889). Zur Lehre von der merfachen Schwangerschaft. (Thesis, Friedrich-Wilhelms Universität zu Berlin). Buchdruckerei von Gustav Shade. Berlin 1889.

Veit, G. (1855). Beiträge zur geburtshülflichen statistik. Monatsschrift für Geburtskunde und Frauenkrankheiten. Bd 6 Heft, 2, 101-132.

Westergaard, H. (1932). Contribution to the history of statistics. London: King (reprinted New York, 1969).

Zeleny, C. (1921). The relative numbers of twins and triplets. Science, 53, 262-263. 